\title{
VOWL 2: User-Oriented Visualization of Ontologies
}

\author{
Steffen Lohmann ${ }^{1}$, Stefan Negru ${ }^{2}$, Florian Haag $^{1}$, and Thomas Ertl ${ }^{1}$ \\ ${ }^{1}$ Institute for Visualization and Interactive Systems (VIS), \\ University of Stuttgart, Universitätsstr. 38, 70569 Stuttgart, Germany \\ \{steffen.lohmann, florian.haag, thomas.ertl\}@vis.uni-stuttgart. de \\ ${ }^{2}$ Faculty of Computer Science, Alexandru Ioan Cuza University \\ Strada General Henri Mathias Berthelot 16, Iasi, Romania \\ stefan.negrueinfo.uaic.ro
}

\begin{abstract}
Ontologies become increasingly important as a means to structure and organize information. This requires methods and tools that enable not only ontology experts but also other user groups to work with ontologies and related structures. We have developed VOWL, a comprehensive and well-specified visual language for the user-oriented visualization of ontologies, and conducted a comparative study on an initial version of VOWL. Based upon results from that study, as well as an extensive review of other ontology visualizations, we have reworked many parts of VOWL. In this paper, we present the new version VOWL 2 and describe how the initial definitions were used to systematically redefine the visual notation. Beside the novelties of the language definition, which is based on a well-defined set of graphical primitives and an abstract color scheme, we briefly describe two implementations of VOWL 2. To gather some insight into the user experience with the new version of VOWL, we have conducted a small qualitative evaluation. We report on the study and its results, which confirmed that not only the general ideas of VOWL but also various of our enhancements for VOWL 2 can be understood by casual ontology users.
\end{abstract}

Keywords: Ontology, visualization, owl, vowl, visual language, semantic web, prefuse, d3, user study.

\section{Introduction}

Ontologies describe the concepts and relationships in an area of knowledge using a logic-based language allowing for automated reasoning. They are no longer exclusively used by ontology experts but also by non-expert users in various domains. However, especially these casual users often have difficulties understanding ontologies.

Ontology visualizations can help in this regard by assisting in the exploration, verification, and sensemaking of ontologies $[15,26]$. They can be particularly useful for casual users, but can also give expert users a new perspective on the ontology. While several visualizations for ontologies have been developed in the last couple of years, they either focus on specific aspects of ontologies or are hard to read for casual users. Furthermore, many visualizations are tailored for a specific task or use special types of diagrams that must first be learned to understand the visualization. 
In order to fill this gap and provide a more intuitive and user-oriented visualization for ontologies, we developed the Visual Notation for OWL Ontologies (VOWL). An early version of VOWL [34] has been compared to the UML-based visualization of ontologies [33]. Based on insights from the comparison, we have completely reworked the notation and developed version 2 with significant improvements and more precise mappings to OWL. One of the main goals of VOWL 2 is to define a visual language that can also be understood by casual users with only little training. In this paper, we present the considerations and concepts of VOWL 2 in detail. Two implementations of VOWL 2 demonstrate its applicability and usability. In addition, we report on a user study that compares VOWL 2 with the two related ontology visualizations GrOWL [24] and SOVA [25].

\section{Related Work}

Quite a number of visualizations for ontologies have been presented in the last couple of years $[10,15,23]$. While some of them are implemented as standalone applications, most are provided as plugins for ontology editors such as Protégé [3].

\subsection{Graph Visualizations of Ontologies}

Many approaches visualize ontologies as graphs, which is a natural way to depict the concepts and relationships in a domain of knowledge. The graphs are often rendered in force-directed or hierarchical layouts, resulting in appealing visualizations. However, only few visualizations show the complete ontology, but most focus on certain parts of it. For instance, OWLViz [21], OntoTrack [27], and KC-Viz [31] depict only the class hierarchy of ontologies. OWLPropViz [37], OntoGraf [11], and FlexViz [12] represent different types of property relations, but do not show datatype properties and property characteristics required to fully understand ontologies.

A smaller number of works provide more comprehensive graph visualizations that represent all key elements of ontologies. Unfortunately, the different ontology elements are often hard to distinguish in the visualizations. For instance, TGViz [5] and NavigOWL [22] use very simple graph visualizations where all nodes and links look the same except for their color. This is different in GrOWL [24] and SOVA [25], which define more elaborated notations using different symbols, colors, and node shapes. However, as the notations of both visualizations rely on symbols from description logic [6], they are not perfectly suited for casual users. In addition, some details important for understanding ontologies, such as property labels and characteristics, are not included in the visualizations.

Other graph visualizations focus on specific tasks. For instance, the RelFinder [18] visualizes relationships between individuals described by ontologies and makes these relationships interactively explorable. GLOW uses a radial tree layout and hierarchical edge bundles to depict ontologies [20]. Both approaches provide some insight into links between certain classes and individuals, but they do not give an overview over the complete ontology. 


\subsection{Ontology Visualizations based on Specific Diagram Types}

There are also a number of works that apply other diagram types than graph visualizations to represent ontologies. For instance, Jambalaya [36] and OWL-VisMod [14] use treemaps to depict the class hierarchy of ontologies. Jambalaya additionally provides a nested graph visualization called SHriMP that allows to split up the class hierarchy into different views [36]. CropCircles is a related visualization technique that visualizes the class hierarchy of ontologies and supports the identification of "undermodeled" ontology parts [38]. All these approaches visualize mainly the class hierarchy, without considering other property relations.

Cluster Maps are a visualization based on nested circles that has also been successfully applied to ontologies [13]. Instead of showing the class hierarchy, Cluster Maps visualize individuals grouped by the classes they are instances of. Similar techniques are used in VisCover [28] and OOBIAN Insight [2] that offer a number of interactive filtering capabilities. Also related is the approach of gFacet [17], where individuals are grouped by their classes and can be filtered by selecting linked individuals or data values. While being appealing visualizations, they show only a selection of classes along with their instances but do not provide a complete visualization of the ontology. Another related approach is OntoTrix [7], which represents ontologies with the NodeTrix visualization technique, a combination of node-link diagrams and adjacency matrices [19]. While it also focuses on the visualization of individuals and their connections within the ontology, it provides a more complete image of the ontology. However, the ontology is converted into the NodeTrix structure for the visualization, making it difficult to get an idea of its global structure and topology.

A powerful type of diagram related to OWL and often reused to visualize ontologies is the class diagram of the Unified Modeling Language (UML) [4]. Precise mappings between OWL and UML class diagrams have been specified in the Ontology Definition Metamodel (ODM) [1], among others. A major drawback of these attempts is that they require some knowledge about UML class diagrams. While these diagrams are well-known to many people working in IT, people from other domains have difficulties interpreting UML class diagrams correctly, as we found in the aforementioned comparative study [33].

\subsection{Discussion of Related Work}

Looking at the multitude of approaches, some common characteristics stand out: Most visualizations utilize a well-known type of diagram for ontology visualization (graph visualization, treemap, UML), use a $2 \mathrm{D}$ representation and focus on specific aspects of the ontologies. Only few attempts aim for a comprehensive ontology visualization. Even less approaches provide an explicit description of the visual notation, i.e. a specification that clearly defines the semantics of the graphical elements. Often, there is no clear visual distinction between different property types or between classes, properties, and individuals.

In addition, many works implement a stepwise approach of ontology exploration, where only a root class is shown at the beginning and the user has to navigate through the visualization. We rather aim for an approach that provides users with an overview of 
the complete ontology and let them subsequently explore parts of it in depth, following the popular information seeking mantra of "overview first, zoom and filter, then detailson-demand" [35]. We chose this approach as we consider it important to give the users a visual impression of the size and topology of the ontology before they start to explore it any further.

Most importantly, we aim for an intuitive visualization that is also comprehensible to users less familiar with ontologies, while most of the related work has rather been designed for ontology experts.

\section{VOWL 2: Visual Notation for OWL Ontologies}

Based upon a review of the related work and our comparative evaluation [33], we decided to retain numerous traits of the initial VOWL version (VOWL 1). As already mentioned, graphs seem to be the natural and intuitive way to represent ontologies, which is confirmed by many of the related approaches reported above. VOWL is based on a mapping of OWL elements to graphical depictions that are combined into a graph representing the ontology. For VOWL 2, we have reworked these mappings and taken into account the exact semantics of all definitions from OWL that were considered. In particular, we have broken down the components of VOWL to a set of basic building blocks consisting of shapes and colors that express specific aspects of the OWL elements (datatype or object properties, different class and property characteristics, etc.), also taking into account any possible combinations thereof.

VOWL 1 explicitly included an integrated view that would display the TBox of an ontology along with information from the ABox. Comments from the initial user study on VOWL 1, however, led us to conclude that concerns about the scalability of the integrated view were justified. Even with few instances per class, additional information such as property values of instances would be difficult to show without creating lots of clutter. Therefore, VOWL 2 focuses on displaying primarily the TBox and only optionally integrates some ABox information in the visualization itself.

\subsection{Basic Building Blocks of VOWL}

The basic building blocks of VOWL 2 are a clearly defined set of graphical primitives and a color schema. In addition, VOWL 2 uses a force-directed graph layout along with splitting rules that specify which elements are multiplied in the visualization.

Graphical Primitives VOWL 1 defined graphical symbols for a number of OWL concepts. For VOWL 2, we took into account those definitions, but based the visualization upon a more abstract and systematic approach. The alphabet of the visual language is now formed by only a handful of graphical primitives and features. Table 1(a) lists these primitives and the ontology elements they are applied to.

As the depiction of single instances was not considered a crucial part, classes are simply depicted as a circle in VOWL 2, without any additional elements to accommodate instance information. Where available and desired, the number of instances may still be implied by modifying the radius of the circle compared to the default radius. 
VOWL 2 does not specify a particular scaling method for the circle sizes, but good results will likely be achieved with a logarithmic or square-root scaling in most cases. The class representation of owl: Thing has a fixed size, as it usually does not carry any particular domain information and as all individuals in an ontology are instances of owl: Thing according to the OWL specification.

Like in VOWL 1, property relations are expressed by labeled arrows. The labels do not have arrowheads any more, as the user study revealed that their intended direction was often ambiguous, particularly for vertical edges. Other edges are completely left out and replaced by additional texts in the labels or interactive features to reduce the number of crossing edges, such as inheritance relations for subproperties.

After reviewing the related work, we realized that the use of description logic and other symbols to express concepts such as the union or the intersection of classes can be unnecessarily confusing to lay users. Therefore, VOWL 2 combines or replaces these symbols with graphical representations reminiscent of Venn diagrams to more clearly communicate the underlying set operation.

Table 1. Graphical primitives and color scheme that form the basic building blocks of VOWL.

(a) Graphical primitives

\begin{tabular}{|c|c|}
\hline Primitive & Application \\
\hline O & classes \\
\hline 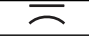 & properties \\
\hline 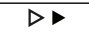 & property directions \\
\hline ] & datatypes, property labels \\
\hline (....... & special classes/properties \\
\hline $\begin{array}{l}\text { text } \\
\text { number } \\
\text { symbor }\end{array}$ & labels, cardinalities \\
\hline
\end{tabular}

(b) Excerpt of the VOWL color scheme

\begin{tabular}{|l|l|l|}
\hline Name & Color & Application \\
\hline General & & classes, object properties, disjoint relations \\
\hline Rdf & & elements based upon RDF and RDF schema \\
\hline External & & external classes and properties \\
\hline Deprecated & & deprecated classes and properties \\
\hline Datatype & & datatypes, literals \\
\hline Datatype property & & datatype properties \\
\hline Highlighting & & interactively highlighted elements \\
\hline
\end{tabular}

Color Scheme Since the results of the user study on VOWL 1 showed that different colors are very helpful in identifying the different elements, a stronger focus was placed on creating systematic coloring rules for VOWL 2. We therefore created a color scheme that clearly defines foreground and background colors for all elements based on a number of attributes. While colors in the VOWL 1 specification were statically linked to the visual elements, VOWL 2 defines colors by their function, for example to mark deprecated or external elements compared to the default color of elements such as classes and property labels. Where several of the coloring rules may apply, priority rules are specified.

VOWL 1 and most related work rely on a concrete set of colors or do not specify colors at all. We acknowledged that a specific color scheme may not always be adequate and therefore defined the VOWL 2 color scheme in a more flexible way by using abstract color names, such as the ones shown in Table 1(b). The specification still recommends a particular color mapping, but it is meant merely as a default suggestion and any references to colors in the specification can be treated as variables. The color scheme comes with guidelines on how the colors should relate to each other. This way, as little information as possible is lost even when the visualization is converted to greyscale or viewed by color-blind users. 


\subsection{Visual Elements and Graph Visualization}

The VOWL 2 specification includes the most common visual elements based on the aforementioned primitives and colors. A selection thereof is shown in Figure 1. In several cases, information is indicated slightly redundantly. For example, equivalent classes both carry a double border as well as several class names in their label, and external classes both adhere to the external color as defined in the color scheme and sport the hint "external" below their name. While the colors improve the overview in most situations, the additional texts ensure that even in the absence of colors, the important aspects of the ontology are still intelligible. Moreover, we wanted to make the visualization more self-explanatory with the text representations. Similar to the conflicting colors, the specification contains guidelines on how to combine several of the additional text markers.
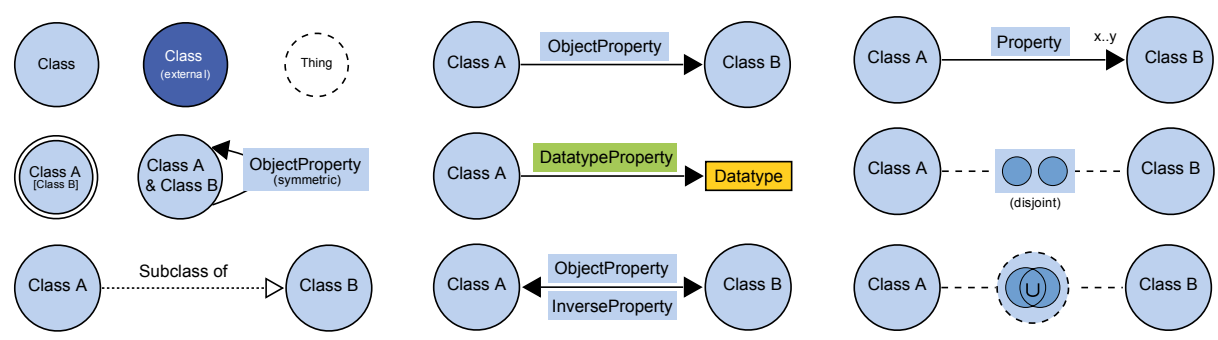

Fig. 1. A selection of visual elements from the VOWL 2 specification.

As participants of the VOWL 1 user study stated the desire for an interactive highlighting of certain information and relationships, some of the visual element definitions now include guidelines on how to design interactive implementations. For example, while the aforementioned inheritance relationship between subproperties is not expressed by an arrow any more in VOWL 2, hovering over a subproperty is defined to automatically highlight its parent property and vice-versa in an interactive context. ${ }^{1}$

The visual elements are combined to a graph representing the entire ontology. Such VOWL graphs generally use a force-directed layouting mechanism. In order to relax the energy of the force-directed layout and reduce the visual importance of certain very frequently referred to, but very generic ontology elements, some elements can be multiplied to appear more than once in a VOWL graph. While this was already the case for owl: Thing in VOWL 1, for VOWL 1 we have systematically defined generic splitting rules. These rules determine that there may be no multiplication for elements, multiplication across the whole graph, or multiplication for each connected class. The VOWL 2 specification relies on those rules to define the splitting of ow 1 : Thing into several representations, as well as that of certain other elements such as datatypes or literals.

\footnotetext{
${ }^{1}$ The graphical representations of further OWL elements are defined in the VOWL 2 specification that is publicly available on the web at the persistent URL http://purl.org/vowl. Most of the remaining elements are variations of the ones presented in Figure 1, such as additional property attributes (e.g. functional, transitive) and further set operators.
} 
Figure 2 shows the graph visualizations resulting for two smaller ontologies if the visual elements are rendered and combined according to the VOWL 2 specification. In this case, the Modular Unified Tagging Ontology (MUTO) and the Friend of a Friend (FOAF) vocabulary are depicted, which contain several of the visual elements described above.

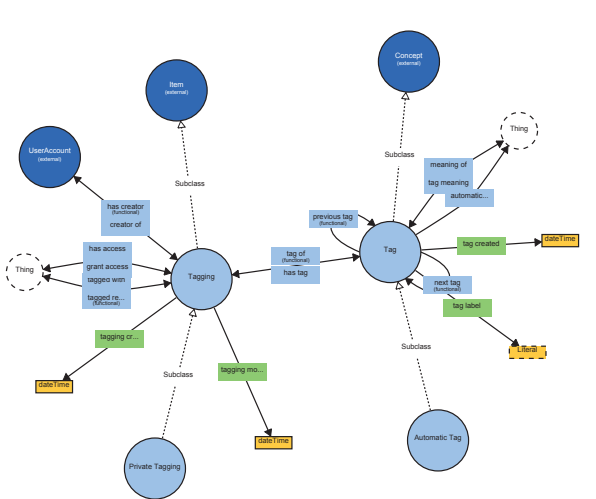

(a) MUTO (version 1.0)

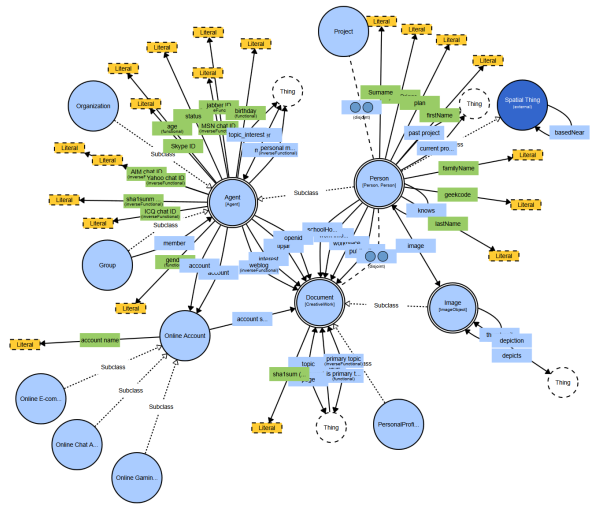

(b) FOAF (version 0.99)

Fig. 2. Overall visual impression of two small ontologies visualized with WebVOWL.

\subsection{Implementations of VOWL 2}

We have implemented VOWL 2 in two different tools that demonstrate its applicability and usability, ProtégéVOWL and WebVOWL. ProtégéVOWL is a Java-based plugin for the aforementioned ontology editor Protégé that makes use of the visualization toolkit Prefuse [16]. ${ }^{2}$ It implements the VOWL 2 specification to a large extent and uses the data model supplied by the underlying Protégé API. WebVOWL is a standalone application based on web technologies and the visualization library D3 [8]. Instead of implementing an OWL parser itself, it specifies a JSON structure that the ontology needs to be transformed into. This makes WebVOWL independent from a particular OWL parser and broadly applicable. In addition, WebVOWL allows to export the complete ontology visualization or a portion of it as SVG image that can be opened in other programs, edited, shared, and printed.

Both tools use a physics simulation to generate the force-directed graph layout provided by the visualization toolkits. The forces are iteratively applied in this simulation resulting in an animation that dynamically positions the nodes. The users can smoothly zoom in to explore certain ontology parts in detail or zoom out to analyze the global structure of the ontology. They can pan the background and move elements around, which results in a repositioning of the nodes by an animated adaptation of the forcedirected layout. Both implementations support interactive highlighting and display ad-

\footnotetext{
${ }^{2}$ A demo of ProtégéVOWL has been presented at ESWC 2014 [30].
} 
ditional ontology-related information on the selected elements on demand to complement the visualization. Moreover, interaction with the force-directed layout is provided, as the attraction forces between nodes can be modified and the automatic layout can be suspended in favor of a manual repositioning of nodes. Since datatypes have a separate attraction force, they can be placed in close proximity to the classes they are connected with, to emphasize their radial arrangement and further increase readability.

The user interfaces of both tools consist of three parts: A viewer displaying the ontology visualization, a sidebar listing details about the selected elements, and the controls allowing to adapt the force-directed graph layout. We developed the tools so that they work in different environments, including settings with touch interaction. For instance, zooming can not only be performed with a mouse wheel but also with a double click/touch or two fingers zooming gestures on the canvas. As some features, such as mouseover effects, may not be available in all environments (e.g. in touch interfaces), we took care that they are not crucial for the interaction or for understanding the ontology. Both tools are released under the MIT license and are available for download at http://vowl.visualdataweb.org.

To the best of our knowledge, WebVOWL is the first tool for comprehensive ontology visualization that is completely based on open web standards. Related tools running in web browsers, such as FlexViz [12] or OOBIAN Insight [2], are implemented with technologies like Adobe Flex or Microsoft Silverlight that require proprietary browser plugins. It is our hope that WebVOWL will be useful to researchers and developers and integrated with frameworks and tools for ontology processing, editing and validation in the future.

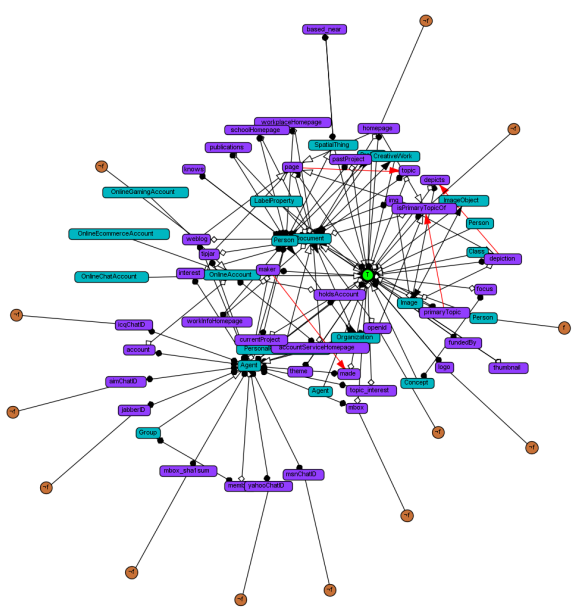

(a) SOVA

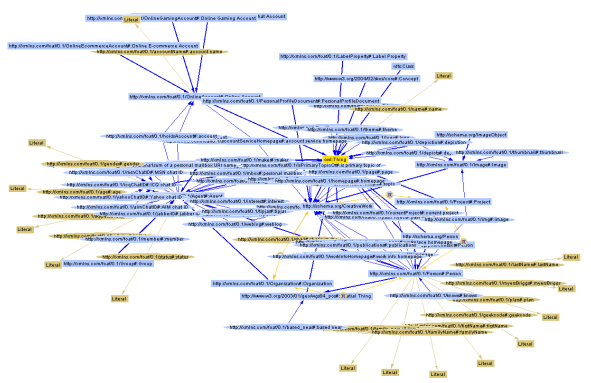

(b) GrOWL

Fig. 3. Overall visual impression of the FOAF ontology (version 0.99) visualized with two of the three approaches compared in the user study. The FOAF ontology was one of the three ontologies presented in the study and one of the two ontologies used in the study tasks. 


\section{Evaluation}

We evaluated how well users could handle VOWL by comparing it to the ontology visualizations SOVA [25] and GrOWL [24]. We chose those two, as they can both be used to gain a general overview over ontologies. Also, they are based on a systematic mapping between ontology concepts and graphical elements. This makes them most related in purpose and scope to VOWL (cf. Section 2), even though both SOVA and GrOWL include some formal symbols. The VOWL visualization was shown in WebVOWL, as it fully implements the VOWL 2 specification, which was not the case for ProtégéVOWL at the time of the study.

In the user study, we presented three ontologies to the participants: Questions were asked about the FOAF ontology [9] (version 0.99) as an example of a smaller ontology, and the Personas Ontology [32] (version 1.5) as an example of a more extensive one. In addition, we included MUTO [29] (version 1.0) as a small training ontology, to give participants an opportunity to familiarize themselves with each visualization approach and the user interface of the respective implementation. The FOAF ontology visualized by SOVA and GrOWL is shown in Figure 3, the VOWL representation is depicted in Figure 2(b).

\subsection{Tasks}

We prepared eighteen comprehension tasks. All tasks referred to structural attributes that someone trying to understand an ontology would have to recognize, such as "Which classes appear to be crucial for the ontology?", "Which data properties does the class Document have?", or "Can you find any classes that serve as a generalization of several subclasses?". The tasks reflected questions that users working with an ontology would have to pose in order to identify, use or edit parts of ontologies. The tasks also forced study subjects to analyze the visualizations and recognize relationships between ontology elements, just like actual users of ontologies would have to.

While we did check the correctness of the answers, we were primarily interested in the comments made by the participants during task completion. Therefore, we asked participants to adhere to the "think-aloud" method and state everything they were thinking, feeling, considering, or doing with respect to the visualizations.

\subsection{Materials}

In total, we prepared 18 tasks, six per visualization, and among those, three for each of the two ontologies (FOAF and Personas Ontology). We also prepared a brief printed introduction to the topic of ontologies. It included a quick overview over the concepts relevant to the study comprising of classes, properties, subclassing, equivalent classes, set operators, and imported ontology elements. Moreover, we printed a table for each of the evaluated visualizations, showing how the concepts are depicted in the given notations and how a very small exemplary ontology that combines several of the elements looks like. Finally, a small questionnaire about the experienced ease-of-use was prepared for each visualization, and an additional questionnaire asked for the prior knowledge of the participants. 
Implementations of the three visualizations-the SOVA plugin for Protégé, the GrOWL Java application, and WebVOWL in a Mozilla Firefox browser-were installed and running on the test computer. In the case of SOVA, we used the plugin version 0.8.1, which was the latest available version at the time of conducting the user study. Unfortunately, that version did not support the display of datatype properties, which is why tasks related to datatype properties in SOVA had to be skipped. The three ontologies (MUTO, FOAF, and Personas) were loaded in each of the visualization applications and displayed on the 24 " TFT monitor at a resolution of $1920 \times 1200$ pixels.

\subsection{Participants}

We chose to conduct the study with participants who may have to work with ontologies at some point, though not necessarily with formal ontology languages like OWLin short, casual ontology users. We recruited six researchers from various fields of information technology (not including Semantic Web technologies) between 29 and 57 years of age. All participants were roughly familiar with the idea of ontologies and/or had some knowledge in related topics, such as object-oriented class structures, UML class diagrams or ER diagrams for database modelling. Two of them had worked with ontologies in the past, but only to a limited extent and without getting in touch with their formal representations.

The tested ontologies were unknown to the study participants. While two participants had already heard about the FOAF project, they had never seen the FOAF ontology before. Another two of the participants had some passing knowledge about the topic of ontology visualization, but none of them had come in contact with any of the three visualizations evaluated in the study.

\subsection{Design and Procedure}

The study had a within-subjects design, so that participants could compare the different visualizations. The presentation of the three visualizations was counterbalanced to avoid order effects. The order of the tasks remained fixed, as some of them would require incremental knowledge. In combination with the counterbalancing of the visualizations, this resulted in a setting where each task had to be solved for each of the visualizations by some of the participants. Likewise, the FOAF ontology was always shown before the Personas Ontology, as the latter has a larger size and is more difficult to grasp.

Participants first had to complete the questionnaire about their prior knowledge before being shown the introduction into ontologies. Meanwhile, it was announced that in the case of any doubts, questions for clarification could be asked at any point before or during the study. After briefly familiarizing themselves with the relevant concepts, participants had to use the three visualizations, one at a time.

For each visualization, participants were first provided with the printed table explaining the notation and had an opportunity to explore the MUTO ontology on screen. When they felt sufficiently familiar with the visualization, they had to perform the three tasks for each of the two ontologies, i.e. for FOAF and the Personas Ontology. The study supervisor would note down any of the participants' statements as well as the solutions they arrived at. 
After completing the tasks for all three visualizations, participants received the questionnaires about the visualizations. On those questionnaires, they had to rate each visualization by the criteria of clarity, learnability, ease of finding elements, mappings between visual and conceptual elements, use of colors and use of shapes.

\subsection{Results}

In general, participants could solve most of the tasks correctly (84\%). Two participants gave up solving some of the tasks in GrOWL, as the implementation lacked a feature to search for specific elements and the participants could not find these elements by visually scanning the graph.

Comments were generally favorable toward VOWL ("properties and classes can be distinguished very well", "not so much tangle and less edges [compared to the others]"), which was especially praised for the clear visual distinction of classes, properties and other ontology elements. Several participants pointed out that classes could easily be recognized due to their different shape and size compared to other graphical elements. The textual labels indicating the meaning of various VOWL elements were mostly thought of as beneficial; one user even stated that there was no need to use the printed table as the VOWL visualization was very self-explanatory. On the other hand, one participant with UML knowledge found some of the labels to be unnecessarily cluttering the visualization, such as the subclass text on the UML-inspired class inheritance arrows.

Participants considered the animated force-directed layout in all three visualizations to be beneficial, as nodes would to some extent arrange themselves. Moreover, they sometimes tried to get a clearer understanding of which properties were connected to which classes. One participant remarked that circles are a good choice for representing classes in VOWL, as arrow heads of edges pointing to the circles would align nicely around the circle with minimal overlapping.

Overall, VOWL 2 was assessed to be well readable due to the comparably low number of edges and, in particular, edge crossings. This effect was achieved both by avoiding several edges present in other visualizations, such as between equivalent classes, and by applying the splitting rules for node multiplication. All but one of the participants understood the way equivalent classes were displayed. Two more participants asked for a clarification, as they wondered whether there would be multiple copies of the double-ringed equivalent class circles, one for each equivalent class, or only one in all. As opposed to that, the general base class ow 1 : Thing was indeed displayed several times according to the splitting rules of VOWL 2 (cf. Section 3.2). Interestingly, this was instantly understood and pointed out whenever participants encountered ow 1 : Thing in one of the tasks solved in VOWL. While multiple copies of owl: Thing at first create slightly more nodes, the edges linking to those owl:Thing nodes are overall shorter and with less edge crossings, again contributing to the aforementioned impression that VOWL does not feature a 'maze of edges'.

Many of the other comments dealt with implementation-specific issues. The most important features asked for (when they were missing) or praised (when they were present) were a continuous zooming feature-for which most participants preferred the 
mousewheel, as offered in VOWL and SOVA — and a search facility to quickly find particular classes (or other elements) by name, which was present in SOVA, but available in WebVOWL only in a limited fashion due to the built-in browser text search. Furthermore, a highlighting feature that changes the color of all directly connected nodes upon hovering the mouse cursor over one node, like the one available in SOVA, was stated to be useful. It was, however, noted, that such a highlighting should not completely replace the original node colors.

\section{Discussion and Future Work}

In an earlier effort to create a uniform visual notation for OWL ontologies, we developed a first version of VOWL [34]. Based upon that work, related endeavors, as well as findings from a user study [33], we have redesigned large parts of the notation to create VOWL 2, a visual language that can also be understood by casual ontology users. We have described the key considerations, features and capabilities of VOWL 2 and have presented two implementations of the visualization, a plugin for the widely used ontology editor Protégé and a responsive web application. Moreover, we have reported on a qualitative user study that compared VOWL 2 to two other interactive ontology visualizations to get a better understanding of its readability and usability. We found that users were able to solve the vast majority of the tasks correctly and received overall favorable comments concerning VOWL 2 .

\subsection{Discussion of Study Results}

Structurally, the design of the graph visualization that allowed moving nodes with the pointing device was welcomed. Study participants would frequently use the opportunity to reposition single nodes in the graph manually in order to get a clearer understanding of the connections present. Along with the force-directed layout, most of the graph would align itself automatically according to the users' input. Unfortunately, disjoint subgraphs can get pushed out of sight, so adding some invisible link to create a slight attraction to the main part of the graph still needs to be tackled. Nonetheless, the force-directed layout led to highly connected nodes that could be easily identified, as intended; in this case, the significant, deeply integrated classes of an ontology would clearly stand out. At the same time, the user study revealed that the idea of multiplying general nodes (e.g. owl:Thing or rdfs:Literal) so they do not appear overly important can be understood by users.

Another factor that gives the graph visualization a cleaner appearance is the unification of equivalent nodes. Although the equivalence relationship was correctly recognized in the user study, several users were unsure whether equivalent classes were displayed as several nodes, each referring to the other nodes, or as only one node.

The fact that many elements are explicitly labeled evoked mostly positive feedback among the study participants. One of the users stated that VOWL 2 is so selfexplanatory that no notation reference was needed at all, while other users commented that some of the labels could be removed from the visualization without loss of clarity. Thus, it seems to be advisable to give users a choice whether such labels are displayed. 


\subsection{Open Issues}

The ontologies used in the user study, which were of a relatively moderate size (comprising of approximately 15 to 50 classes), could still be handled in VOWL 2. However, there is no upper limit for the size of ontologies, both because a vast number of topics can be covered in one ontology, and because an ontology can be modeled down to an arbitrary level of detail. Gaph visualizations are, on the other hand, viable only up to a certain graph size, at which point the overview is lost and the graph is not easily usable any more. VOWL 2 mitigates this only slightly based on its splitting rules and the unification of equivalent classes. Ongoing research on VOWL will have to look into means of dealing with this problem, at latest when VOWL is used to analyze the connections across several overlapping ontologies. Solutions will incorporate both considerations on the automatical and manual detection of ontology parts that carry context-specific importance, so parts of the ontology can be temporarily hidden or bundled, as well as general strategies for handling very large graphs used in the field of graph visualization.

A related issue exists due to the fact that ontology elements have no inherent relative location information. Therefore, all elements are initially placed in a random manner in the force-directed layout. While this does not influence a single session of work with a VOWL depiction of an ontology, it prevents the use of a "mental map" by a user who renders an ontology several times with VOWL, as the elements are at different locations every time. Future work will have to develop reasonable guidelines on how to best place ontology elements so their positioning follows a reproducible pattern.

\section{References}

1. Ontology Definition Metamodel. http://www.omg.org/spec/ODM/.

2. OOBIAN Insight. http://dbpedia.oobian.com.

3. Protégé Wiki. http://protegewiki.stanford.edu/wiki/Visualization.

4. Unified Modeling Language. http://www. uml.org.

5. H. Alani. TGVizTab: An ontology visualisation extension for protégé. In 2nd Workshop on Visualizing Information in Knowledge Engineering, VIKE '04, 2003.

6. F. Baader, D. Calvanese, D. L. McGuinness, D. Nardi, and P. F. Patel-Schneider, editors. The Description Logic Handbook: Theory, Implementation, and Applications. Cambridge University Press, 2003.

7. B. Bach, E. Pietriga, I. Liccardi, and G. Legostaev. OntoTrix: a hybrid visualization for populated ontologies. In Proceedings of the 20th International Conference on World Wide Web (Companion Volume), WWW'11, pages 177-180. ACM, 2011.

8. M. Bostock, V. Ogievetsky, and J. Heer. D3 data-driven documents. IEEE Transactions on Visualization and Computer Graphics, 17(12):2301-2309, 2011.

9. D. Brickley and L. Miller. FOAF Vocabulary Specification 0.99. http: / / xmlns . com/foaf / spec/, 2014

10. A.-S. Dadzie and M. Rowe. Approaches to visualizing linked data: A survey. Semantic Web, 2(2):89-124, 2011.

11. S. Falconer. OntoGraf. http://protegewiki.stanford.edu/wiki/OntoGraf, 2010.

12. S. M. Falconer, C. Callendar, and M.-A. Storey. A visualization service for the semantic web. In Proceedings of the 17th International Conference on Knowledge Engineering and Management by the Masses, EKAW'10, pages 554-564. Springer, 2010. 
13. C. Fluit, M. Sabou, and F. van Harmelen. Ontology-based information visualization: Toward semantic web applications. In Visualizing the Semantic Web, pages 36-48. Springer, 2002.

14. F. J. García-Peñalvo, R. Colomo-Palacios, J. García, and R. Therón. Towards an ontology modeling tool. a validation in software engineering scenarios. Expert Systems with Applications, 39(13):11468-11478, 2012.

15. V. Geroimenko and C. Chen. Visualizing the Semantic Web: XML-Based Internet and Information Visualization. Springer, 2nd edition, 2006.

16. J. Heer, S. K. Card, and J. A. Landay. Prefuse: A toolkit for interactive information visualization. In Proceedings of the SIGCHI Conference on Human Factors in Computing Systems, CHI '05, pages 421-430. ACM, 2005.

17. P. Heim, T. Ertl, and J. Ziegler. Facet graphs: Complex semantic querying made easy. In Proceedings of the 7th Extended Semantic Web Conference, Part I, ESWC '10, pages 288302. Springer, 2010.

18. P. Heim, S. Lohmann, and T. Stegemann. Interactive relationship discovery via the semantic web. In Proceedings of the 7th Extended Semantic Web Conference, Part I, ESWC '10, pages 303-317. Springer, 2010.

19. N. Henry, J.-D. Fekete, and M. J. McGuffin. NodeTrix: A hybrid visualization of social networks. IEEE Transactions on Visualization and Computer Graphics, 13(6):1302-1309, 2007.

20. D. Holten. Hierarchical edge bundles: Visualization of adjacency relations in hierarchical data. IEEE Transactions on Visualization and Computer Graphics, 12(5):741-748, Sep. 2006.

21. M. Horridge. OWLViz. http://protegewiki.stanford.edu/wiki/OWLViz, 2010.

22. A. Hussain, K. Latif, A. Rextin, A. Hayat, and M. Alam. Scalable visualization of semantic nets using power-law graphs. Applied Mathematics \& Information Sciences, 8(1):355-367, 2014.

23. A. Katifori, C. Halatsis, G. Lepouras, C. Vassilakis, and E. Giannopoulou. Ontology visualization methods - a survey. ACM Computer Surveys, 39(4), Nov. 2007.

24. S. Krivov, F. Villa, R. Williams, and X. Wu. On visualization of OWL ontologies. In C. J. Baker and K.-H. Cheung, editors, Semantic Web, pages 205-221. Springer, 2007.

25. P. Kunowski and T. Boiński. SOVA - Simple Ontology Visualization API. http:// protegewiki.stanford.edu/wiki/SOVA, 2012.

26. M. Lanzenberger, J. Sampson, and M. Rester. Visualization in ontology tools. In Proceedings of the International Conference on Complex, Intelligent and Software Intensive Systems, CISIS '09, pages 705-711, 2009.

27. T. Liebig and O. Noppens. OntoTrack: A semantic approach for ontology authoring. Web Semantics: Science, Services and Agents on the World Wide Web, 3(2-3):116-131, 2005.

28. T. Liebig, O. Noppens, and F. W. von Henke. Viscover: Visualizing, exploring, and analysing structured data. In Proceedings of the IEEE Symposium on Visual Analytics Science and Technology, VAST '09, pages 259-260. IEEE, 2009.

29. S. Lohmann. Modular unified tagging ontology (MUTO). http://purl.org/muto/core\#, 2011.

30. S. Lohmann, S. Negru, and D. Bold. The protgvowl plugin: Ontology visualization for everyone. In Proceedings of ESWC 2014 Satellite Events. Springer, to appear.

31. E. Motta, P. Mulholland, S. Peroni, M. d'Aquin, J. M. Gomez-Perez, V. Mendez, and F. Zablith. A novel approach to visualizing and navigating ontologies. In Proceedings of the 10th International Conference on the Semantic Web, Volume I, ISWC '11, pages 470486. Springer, 2011.

32. S. Negru. PersonasOnto. http://blankdots.com/open/personasonto.html, 2014. 
33. S. Negru, F. Haag, and S. Lohmann. Towards a unified visual notation for owl ontologies: Insights from a comparative user study. In Proceedings of the 9th International Conference on Semantic Systems, I-SEMANTICS '13, pages 73-80. ACM, 2013.

34. S. Negru and S. Lohmann. A visual notation for the integrated representation of OWL ontologies. In Proceedings of the 9th International Conference on Web Information Systems and Technologies, WEBIST '13, pages 308-315. SciTePress, 2013.

35. B. Shneiderman. The eyes have it: A task by data type taxonomy for information visualizations. In Proceedings of the 1996 IEEE Symposium on Visual Languages, VL '96, pages 336-343. IEEE, 1996.

36. M.-A. Storey, N. F. Noy, M. Musen, C. Best, R. Fergerson, and N. Ernst. Jambalaya: An interactive environment for exploring ontologies. In Proceedings of the 7th International Conference on Intelligent User Interfaces, IUI '02, pages 239-239. ACM, 2002.

37. L. Wachsmann. OWLPropViz. http://protegewiki. stanford.edu/wiki/OWLPropViz, 2008.

38. T. D. Wang and B. Parsia. CropCircles: topology sensitive visualization of OWL class hierarchies. In Proceedings of the 5th International Conference on the Semantic Web, ISWC '06, pages 695-708. Springer, 2006. 